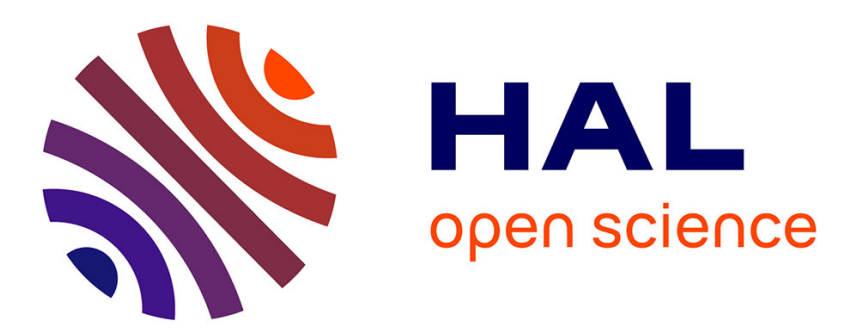

\title{
Finite-time Attractive Ellipsoid Method: Implicit Lyapunov Function Approach
}

\author{
Manuel Mera, Andrey Polyakov, Wilfrid Perruquetti
}

\section{To cite this version:}

Manuel Mera, Andrey Polyakov, Wilfrid Perruquetti. Finite-time Attractive Ellipsoid Method: Implicit Lyapunov Function Approach. International Journal of Control, 2015, 10.1080/00207179.2015.1118660 . hal-01227455

\section{HAL Id: hal-01227455 \\ https://hal.inria.fr/hal-01227455}

Submitted on 11 Nov 2015

HAL is a multi-disciplinary open access archive for the deposit and dissemination of scientific research documents, whether they are published or not. The documents may come from teaching and research institutions in France or abroad, or from public or private research centers.
L'archive ouverte pluridisciplinaire HAL, est destinée au dépôt et à la diffusion de documents scientifiques de niveau recherche, publiés ou non, émanant des établissements d'enseignement et de recherche français ou étrangers, des laboratoires publics ou privés. 


\title{
Finite-time Attractive Ellipsoid Method: Implicit Lyapunov Function Approach
}

\author{
Manuel Mera*, Andrey Polyakov*,** and Wilfrid Perruquetti ${ }^{* *, *}$ \\ (Received 00 Month 20XX; accepted 00 Month 20XX)
}

\begin{abstract}
A finite-time version, based on Implicit Lyapunov Functions (ILF), for the Attractive Ellipsoid Method (AEM) is developed. Based on this, a robust control scheme is presented to ensure finite-time convergence of the solutions of a chain of integrators with bounded output perturbations to a minimal ellipsoidal set. The control parameters are obtained by solving a minimization problem of the "size" of the ellipsoid subject to a set of Linear Matrix Inequalities (LMI's) constraints, and by applying the implicit function theorem. A numerical example is presented to support the implementability of these theoretical results.
\end{abstract}

\section{Introduction}

The modeling and control of dynamical systems subject to bounded perturbations is one of the most relevant problems in control theory, this is because it is unrealistic to expect actual dynamical systems to be free of noise, or that mathematical models represent perfectly, without uncertainties, real systems. For this reason the research done in the robust control area has been extensive.

The minimization of the effects due to external perturbations and uncertainties on the performances of dynamical systems is a well-known area, where several results have been obtained and a considerable amount of different approaches have been developed, such as sliding-mode control, (Shtessel, Edwards, Fridman, \& Levant, 2014) which only works mainly for matched perturbations; $H_{\infty}$ Control (Orlov \& Aguilar, 2014), which usually asks for a vanishing condition on the perturbations; and neural networks (Haykin, 2009), which has some difficult implementability issues. Among these a remarkable one is the AEM (Poznyak, Polyakov, \& Azhmyakov, 2014), which deals with unmatched and non-vanishing perturbations, also the control gain is usually obtained by the solution of a linear minimization problem, this is a minimization problem with a linear objective function and linear constraints, which suggests an advantage on the computational aspect of the controller design and its implementability.

The history of the AEM approach can be tracked down to the late 60's and early 70's, when the characterization of uncertain dynamics by ellipsoidal sets was first introduced in the works of (Schweppe, 1968) and (Bertsekas \& Rhodes, 1971). Then, the use of ellipsoids as estimations of sets guaranteed to contain a significant variable was further developed in (Kurzhanskii, 1977), (Chernousko, 1994), (Polyak, Nazin, Durieu, \& Walter, 2004). The concept of the asymptotically attractive (invariant) ellipsoid as used in this paper was formalized in (Usoro, Schweppe, Wormley, \& Gould, 1981), (Polyak \& Topunov, 2008) for linear systems and later extended to nonlinear systems in (Poznyak, Azhmyakov, \& Mera, 2011), (Mera, Poznyak, Azhmyakov, \& Fridman, 2009) and (Poznyak et al., 2014).

\footnotetext{
* Non-A INRIA-LNE, Parc Scientifique de la Haute Borne 40, avenue Halley Bat.A, Park Plaza, 59650 Villeneuve d'Ascq, France

${ }^{* *}$ CRIStAL (UMR CNRS 9189), Ecole Centrale de Lille, BP 48, Cité Scientifique, 59651 Villeneuve-d'Ascq, France
} 
The AEM (Gonzalez-Garcia, Polyakov, \& Poznyak, 2009) is based on the Lyapunov analysis, so it is natural to use the ILF method, originally presented in (Korobov, 1979), and later revisited in (Adamy \& Flemming, 2004), (Polyakov, Efimov, \& Perruquetti, 2013), to extend this approach and obtain additional features such as finite-time convergence to the ellipsoidal region.

This is a very desirable property, considering that many applications require control algorithms fast enough to guarantee the convergence of system dynamics to a desirable value or set of values in finite-time. The finite-time stabilization (Bhat \& Bernstein, 2000), (Roxin, 1966), (Moulay \& Perruquetti, 2006) is crucial when the transient dynamics are required to end as fast as possible or in a specific time interval, as in robotics and aeronautics. Some examples of this can be found in (Haimo, 1986) and (Orlov, 2005).

The ILF, as presented in (Polyakov et al., 2013), (Polyakov, Efimov, \& Perruquetti, 2015), is used to construct a robust control strategy by using a Lyapunov function defined implicitly in an algebraic equation. The stability analysis then does not require the explicit solution of this equation, instead the stability conditions can be revised directly using the implicit function theorem (see (Courant \& John, 2000)).

For simplicity, the corresponding analysis is based on homogeneity which is a very useful tool for the study of finite-time stability (see (Zubov, 1964), (Levant, 2005), (Perruquetti, Floquet, \& Moulay, 2008)). Specifically, if an asymptotically stable system is homogeneous of negative degree, then it is finite-time stable.

The AEM with the ILF derives in a minimization problem of the size (trace) of the ellipsoid characterizing matrix subject to a set of constraints obtained from the finite-time stability conditions, which can be expressed as a set of LMI's. The implementable control strategy is obtained from the solution of this optimization problem, and the application of a numerical procedure.

The structure of this paper is the following. The system description and the formal problem statement are presented in section 2. The main concepts and definitions for the AEM and ILF are introduced in section 3. The main result, a robust nonlinear feedback control design, as well as a linear feedback control strategy used for comparison are presented in section 4 . In section 5 an iterative algorithm for obtaining the control parameters is proposed and an example is given to illustrate the implementability of this approach. Finally, section 6 contains the concluding remarks.

\section{Problem Statement}

Considering the system

$$
\begin{aligned}
& \dot{x}(t)=A x(t)+b u(t), \\
& y(t)=x(t)+\xi(t), \forall t \geq 0
\end{aligned}
$$

where $x \in \mathbb{R}^{n}$ is the state vector, $u \in \mathbb{R}$ is the control input, $y \in \mathbb{R}^{n}$ is the measurable output, $\xi \in \mathbb{R}^{n}$ is an unknown but bounded and locally measurable perturbation,

$$
\xi(t)^{T} Q \xi(t) \leq 1, \forall t \geq 0
$$


with $Q=Q^{T}>0$,

$$
A=\left(\begin{array}{cccc}
0 & 1 & \cdots & 0 \\
\vdots & \vdots & \ddots & \vdots \\
0 & 0 & \cdots & 1 \\
0 & 0 & \cdots & 0
\end{array}\right), \text { and } b=\left(\begin{array}{c}
0 \\
\vdots \\
0 \\
1
\end{array}\right)
$$

Note that (2) can also be considered almost everywhere.

Usually, a well developed control scheme for system (1) which describes a chain of integrators, can be easily extended to a wider class of systems such as feedback linearizable nonlinear systems with multiple inputs and multiple outputs (Isidori, 1995). Moreover, the consideration of (1) is also motivated because it can be used to model many mechanical and electromechanical applications such as in (Chernousko, Ananevskii, \& Reshmin, 2008) and (Utkin, Guldner, \& Shi, 2009).

The objective of this paper is to design a robust feedback control strategy for the system (1), this feedback control strategy should ensure finite-time convergence of the system solutions to an ellipsoidal region despite having noisy measurements.

The ILF approach is used to develop an extended version of the AEM which includes finite-time convergence to the ellipsoid. The main tool for these two approaches is the Lyapunov function method, which in this case is implicitly defined as a solution of an algebraic equation. The analysis of the ellipsoidal set attractivity does not require the solution of this equation, because it is possible to use the implicit function theorem to check all the required conditions directly from the implicit formulation.

\section{Preliminaries}

\subsection{Finite-Time Attractive Ellipsoid}

Considering the system of the form

$$
\dot{x}(t)=f(x(t), \xi(t)), \forall t \geq 0 \quad x(0)=x_{0}, \quad \xi(0)=\xi_{0} ;
$$

where

- $x(t) \in \mathbb{R}^{n}$ is the state vector,

- $\xi(t) \in \mathbb{R}^{n}$ is an unknown but bounded perturbation vector

$$
\|\xi(t)\| \leq l_{0}, \forall t \geq 0, l_{0} \in \mathbb{R}_{+},
$$

- $f: \mathbb{R}^{n} \rightarrow \mathbb{R}^{n}$ is a nonlinear continuous vector field.

Assume that the following ellipsoid

$$
\mathcal{E}_{x}:=\left\{x \in \mathbb{R}^{n}: x^{T} \tilde{P}^{-1} x \leq 1\right\}, \quad \tilde{P}=\tilde{P}^{T}>0 .
$$

is an invariant set of (3). Additionally we consider the notation, for any $\theta \in \mathbb{R}^{n}$

$$
\|\theta\|_{\mathcal{E}_{x}}:=\inf _{\eta \in \mathcal{E}_{x}}\|\theta-\eta\|,
$$

for distance from a point $\theta$ to a set $\mathcal{E}_{x}$. 
Definition 1: (Poznyak et al., 2014) The set $\mathcal{E}_{x}$, is an asymptotically attractive ellipsoid for the system (3) if $\left\|x\left(t, x_{0}\right)\right\|_{\mathcal{E}_{x}} \rightarrow 0$, as $t \rightarrow \infty$, for any $x_{0} \in \mathbb{R}^{n}$.

The asymptotic attractivity of $\mathcal{E}_{x}$ guarantees convergence of the system solutions, with any initial condition $x_{0}$, to the ellipsoid. However, it is always desirable to have some estimation for the time of convergence to the set. Addressing this, the definition of finite-time attractive ellipsoid is presented.

Definition 2: The set $\mathcal{E}_{x}$ is a finite-time attractive ellipsoid of system (3) if it is an asymptotically attractive ellipsoid, and there exists a function $T$ (called the settling time function): $\mathbb{R}^{n} \backslash \mathcal{E}_{x} \rightarrow \mathbb{R}_{+}$such that

$$
\left\|x\left(t, x_{0}\right)\right\|_{\mathcal{E}_{x}}=0, \quad \forall t \geq T\left(x_{0}\right)
$$

Note that the Definitions 1 and 2 do not ask for stability of the set and only consider the attractivity property. However, in practice the stability of $\mathcal{E}_{x}$ is preferable for additional robustness.

\subsection{Implicit Lyapunov Method for Attractive Ellipsoid}

For the very specific case when $\xi(t)=0, \forall t$, we have:

Theorem 1 [(Polyakov et al., 2013)]: If there exists a continuous function

$$
\begin{aligned}
& G: \mathbb{R}_{+} \times \mathbb{R}^{n} \rightarrow \mathbb{R} \\
& (V, x) \rightarrow G(V, x)
\end{aligned}
$$

satisfying the conditions

C1) $G$ is continuously differentiable outside the origin for all positive $V \in \mathbb{R}_{+}$and for all $x \in \mathbb{R}^{n} \backslash\{0\}$;

C2) for any $x \in \mathbb{R}^{n} \backslash\{0\}$ there exists $V \in \mathbb{R}_{+}$such that

$$
G(V, x)=0
$$

C3) let $\Phi=\left\{(V, x) \in \mathbb{R}_{+} \times \mathbb{R}^{n} \backslash\{0\}: G(V, x)=0\right\}$, then, $\forall(V, x) \in \Phi$

$$
\lim _{\|x\| \rightarrow 0} V=0^{+}, \quad \lim _{V \rightarrow 0}\|x\|=0, \quad \lim _{\|x\| \rightarrow \infty} V=+\infty
$$

C4) the inequality $\frac{\partial G(V, x)}{\partial V}<0$ holds for all $V \in \mathbb{R}_{+}$and for all $x \in \mathbb{R}^{n} \backslash\{0\}$;

C5) $\frac{\partial G(V, x)}{\partial x} f(x, 0)<0$ holds for all $(V, x) \in \Phi$;

then the origin of (3) is globally uniformly asymptotically stable.

Theorem 2 [(Polyakov et al., 2013)]: If there exists a continuous function $G: \mathbb{R}_{+} \times \mathbb{R}^{n} \rightarrow \mathbb{R}$ that satisfies the conditions C1)-C4) of theorem 1 and the condition

C5bis) there exist $c>0$ and $0<\mu<1$ such that $\frac{\partial G(V, x)}{\partial x} f(x, 0) \leq c V^{1-\mu} \frac{\partial G(V, x)}{\partial V}$ for $(V, x) \in \Phi$, then the origin of the system (3) is globally uniformly finite-time stable and the settling time function satisfies $T\left(x_{0}\right) \leq \frac{V_{0}^{\mu}}{c \mu}$, where $G\left(V_{0}, x_{0}\right)=0$. 
This theorem can be derived from Theorem 1.1 in (Korobov, 2007), (Korobov, 1979) using the implicit function theorem.

Now considering the more general case when $\|\xi(t)\| \leq l_{0}, \forall t \geq 0$ in (3), it is unrealistic to expect that all system trajectories converge to the origin. Still, it is possible for the system trajectories to converge to a certain set depending on $l_{0}$, the bound of the perturbations. In order to define this set in terms of the conditions given by the ILF we present the next Corollary.

Corollary 1: Let the conditions C1)-C4) from theorem 1 hold. If the inequality $\frac{\partial G(V, x)}{\partial x} f(x, \xi)<0$ holds for all $(V, x) \in \Phi$ and all $\|\xi\| \leq l_{0}$ such that $V(x)>1$, then the set

$$
\mathcal{W}:=\left\{x \in \mathbb{R}^{n}:(V, x) \in \Phi, 0<V \leq 1\right\} \cup\{0\},
$$

is globally asymptotically stable for the system (3).

These conditions repeat in an implicit form the requirements for the Lyapunov-like (storage) function of the AEM, this is a positive function which is not necessarily monotonically non-increasing in all $\mathbb{R}^{n}$, but only outside of a bounded set. Conditions $\mathrm{C} 1, \mathrm{C} 2, \mathrm{C} 4$ and the implicit function theorem (Courant \& John, 2000), denote that the equation $G(V, x)=0$ defines a unique function $V: \mathbb{R}^{n} \backslash\{0\} \rightarrow \mathbb{R}_{+}$for all $x \in \mathbb{R}^{n} \backslash\{0\}$. The function $V$ is continuously differentiable outside the origin and $\frac{\partial V}{\partial x}=-\left[\frac{\partial G(V, x)}{\partial V}\right]^{-1} \frac{\partial G(V, x)}{\partial x}$ for $G(V, x)=0, \forall x \neq 0$. The implicit function theorem and conditions C3, C4 and Corollary 1 prove that $\dot{V}<0$, while $V>1$.

The next corollary specifies the finite-time attractive set.

Corollary 2: If there exists a continuous function $G: \mathbb{R}_{+} \times \mathbb{R}^{n}$ that satisfies the conditions $C 1$ - $C 4$, the one in Corollary 1, and additionally it fulfills that there exists $c>0$ and $\mu \in(0,1)$ such that $\frac{\partial G(V, x)}{\partial x} f(x, \xi) \leq c V^{1-\mu} \frac{\partial G(V, x)}{\partial V}$ for $(V, x) \in \Phi,\|\xi\| \leq l_{0}$ and $V(x)>1$, then $\mathcal{W}$ is globally finite-time attractive for the system (3), and the settling time function estimation satisfies $T\left(x_{0}\right) \leq \frac{V_{0}^{\mu}-1}{c \mu}$ with $G\left(V_{0}, x_{0}\right)=0$.

One of the main features of the control design using the AEM is the possibility to minimize in certain sense the ellipsoid size, insuring that the system solutions converge to a minimal set in $\mathbb{R}^{n}$ regardless of perturbations or uncertainties (Poznyak et al., 2014). The usual characterization of the size of the ellipsoid in the AEM is conveniently described by the trace of the matrix $\tilde{P}$. The "natural" restrictions of the system and its parameters are written in the form of LMI's. Hence, the control parameters that ensure convergence to this minimal set are obtained through the solution of a minimization problem which objective function is $\operatorname{tr}(\tilde{P})$, and the corresponding constraints are a set of LMI's. The same approach is used in the next sections to obtain a characterization of the minimal finite-time version of the Attractive Ellipsoid.

\section{Control Design}

\subsection{Finite-time attractive set}

Let us consider the following ILF candidate (Polyakov et al., 2013)

$$
G(V, x):=x^{T} D_{r}\left(V^{-1}\right) P D_{r}\left(V^{-1}\right) x-1,
$$

where $V \in \mathbb{R}_{+}, x \in \mathbb{R}^{n}, P=P^{T} \in \mathbb{R}^{n \times n}, P>0$,

$$
D_{r}(\lambda):=\operatorname{diag}\left\{\lambda^{r_{i}}\right\}
$$


with

$$
r_{i}=1+(n-i) \mu, \quad i \in\{1, \ldots, n\}, \quad 0<\mu<1 .
$$

Denoting

$$
H_{\mu}:=\operatorname{diag}\left\{r_{i}\right\}
$$

and defining $V_{y}:=V(y)$.

Note that when $\mu=0$ equation (5) coincides with the storage function in the AEM.

Theorem 3: Let $\mu \in(0,1), \delta>1, \beta \in \mathbb{R}_{+}$, the matrix

$$
\Omega=\left(\begin{array}{cccc}
A X+b Y+X A^{T}+Y^{T} b^{T} & b Y & b Y & \beta X \\
+H_{\mu} X+X H_{\mu} & -\underline{\gamma}^{-2+2 \mu} \delta X & 0 & 0 \\
Y^{T} b^{T} & 0 & -\beta X & 0 \\
Y^{T} b^{T} & 0 & 0 & -r_{1} \beta \tilde{D}_{m}^{-2}
\end{array}\right)
$$

where $X=X^{T} \in \mathbb{R}^{n \times n}, Y \in \mathbb{R}^{1 \times n}$ and $\tilde{D}_{m} \in \mathbb{R}^{n \times n}$ is a diagonal matrix which $i$-th entry $m_{i}$ is given by

$$
m_{i}:=\max \left\{\left|\underline{\gamma}^{-(n-i+1) \mu}-1\right|,\left|\bar{\gamma}^{-(n-i+1) \mu}-1\right|\right\},
$$

for $i \in\{1, \ldots, n\}$, and

$$
\underline{\gamma}=\sqrt{\frac{r_{1}}{r_{2}}}\left(1-\sqrt{\frac{r_{1}}{r_{3}}}\right), \bar{\gamma}=\sqrt{\frac{r_{2}}{r_{1}}}\left(1+\sqrt{\frac{r_{1}}{r_{3}}}\right),
$$

with $r_{1}:=1 / \lambda_{\max }(X), r_{2}:=1 / \lambda_{\min }(X)$, and $r_{3}:=\lambda_{\min }(Q)$, where $\lambda_{\min }(\cdot)$ and $\lambda_{\max }(\cdot)$ are the minimum and maximum eigenvalues respectively. If the LMI system

$$
\left\{\begin{array}{l}
0<Q^{-1} \leq X \\
0<\delta^{2} X \leq X H_{\mu}+H_{\mu} X \\
0<Q H_{\mu}+H_{\mu} Q \\
\Omega \leq 0
\end{array}\right.
$$

is feasible for some $X$ and $Y$; the control input

$$
u\left(V_{y}, y\right)=V_{y}^{1-\mu} k D_{r}\left(V_{y}^{-1}\right) y
$$

where $k:=Y X^{-1}, V_{y} \in \mathbb{R}_{+}$is such that $G\left(V_{y}, y\right)=0$, with $G$ defined as in (5).

Then the ellipsoid (4), with $\tilde{P}=D_{r}(\bar{\gamma}) X D_{r}(\bar{\gamma})$ is a finite-time attractive ellipsoid for the closedloop system (1) with the settling time function estimation given by $T\left(x_{0}\right) \leq \frac{1}{1-\delta^{-1}} \bar{\gamma}^{\mu}\left(V^{\mu}\left(y_{0}\right)-1\right)$. 


\subsection{Minimization of the Attractive Ellipsoid}

Let us consider the minimization of the ellipsoidal set described in theorem 3. In order to do so we minimize the trace of the matrix $X$ subject to the constraints defined in the LMI's system, as usual. Note that the minimization of $X$ is equivalent to the minimization of the ellipsoidal set characterized by $D_{r}\left(\bar{\gamma}^{-1}\right) P D_{r}\left(\bar{\gamma}^{-1}\right)$. The minimization problem statement is the following

$$
\operatorname{tr}(X) \rightarrow \min _{X, Y, \delta, \beta}
$$

subject to $(6)$

If $\hat{X}$ and $\hat{Y}$ are the solution of the above minimization problem, then control (7) applied to system (1) has the gain $\hat{k}=\hat{Y} \hat{X}^{-1}$.

\subsection{On the boundedness of the control}

If it is necessary to restrict the magnitude of the control signal inside the ellipsoid, it is sufficient to include an additional constraint

$$
\sigma^{-2} k^{T} k \leq P
$$

to the optimization problem (8). This, in terms of the matrices $X$ and $Y$ and using the Schur's complement can be represented in LMI form as

$$
\left(\begin{array}{cc}
X & Y^{T} \\
Y & \sigma^{2}
\end{array}\right) \geq 0
$$

this inequality implies that for any trajectory inside the ellipsoid $\left(V_{y} \leq 1\right)$ we will have

$$
\begin{aligned}
\|u\|^{2} & =V_{y}^{2-2 \mu} y^{T} D_{r}\left(V_{y}^{-1}\right) k^{T} k D_{r}\left(V_{y}^{-1}\right) y \\
& \leq \sigma^{2} V_{y}^{2-2 \mu} y^{T} D_{r}\left(V_{y}^{-1}\right) P D_{r}\left(V_{y}^{-1}\right) y \leq \sigma^{2} V_{y}^{2-2 \mu} \leq \sigma^{2} .
\end{aligned}
$$

\subsection{AEM Linear Case}

We consider a linear version of the AEM, based on (Poznyak et al., 2014) as a frame of reference to weigh the results and the implementability of the extended version presented in this paper. The linear AEM formulation consists in using a feedback $u=k_{c} y$, where $k_{c} \in \mathbb{R}^{1 \times n}$ is a constant gain for system (1), and selecting a quadratic storage function

$$
V_{c}=x^{T} P_{c}^{-1} x
$$

where $Q^{-1} \leq P_{c}=P_{c}^{T} \in \mathbb{R}^{n}$. Defining the vector

$$
z_{c}=\left(\begin{array}{l}
x \\
\xi
\end{array}\right)
$$


the time derivative of the function $V_{c}$ can be written as

$$
\begin{aligned}
& \dot{V}_{c}=2 x^{T} P_{c}^{-1}\left[A x+b k_{c}(x+\xi)\right]= \\
& z_{c}^{T}\left(\begin{array}{cc}
P_{c}^{-1} A+A^{T} P_{c}^{-1}+P_{c}^{-1} b k_{c}+k_{c} b P_{c}^{-1} & P_{c}^{-1} b k_{c} \\
k_{c}^{T} b^{T} P_{c}^{-1} & 0
\end{array}\right) z_{c}
\end{aligned}
$$

adding and subtracting $\alpha_{c} x^{T} P_{c}^{-1} x$ and $\alpha_{c} \xi^{T} Q \xi$, with $\alpha_{c} \in \mathbb{R}_{+}$we obtain

$$
\dot{V}_{c}=z_{c}^{T} W_{0} z_{c}-\alpha_{c} x^{T} P_{c}^{-1} x+\alpha_{c} \xi^{T} Q \xi
$$

where

$$
W_{0}:=\left(\begin{array}{cc}
P_{c}^{-1} A+A^{T} P_{c}^{-1}+P_{c}^{-1} b k_{c}+k_{c} b P_{c}^{-1}+\alpha_{c} P_{c}^{-1} & P_{c}^{-1} b k_{c} \\
k_{c}^{T} b^{T} P_{c}^{-1} & -\alpha_{c} Q
\end{array}\right),
$$

introducing $\delta_{c} \in \mathbb{R}_{+}$such that $\alpha_{c} Q \geq \delta_{c} P_{c}^{-1}$, the time derivative of $V_{c}$ can be upper bounded as

$$
\dot{V}_{c} \leq z_{c}^{T} W_{1} z_{c}-\alpha_{c} V_{c}+\alpha_{c}
$$

with

$$
W_{1}:=\left(\begin{array}{cc}
P_{c}^{-1} A+A^{T} P_{c}^{-1}+P_{c}^{-1} b k_{c}+k_{c} b P_{c}^{-1}+\alpha_{c} P_{c}^{-1} & P_{c}^{-1} b k_{c} \\
k_{c}^{T} b^{T} P_{c}^{-1} & -\delta_{c} P_{c}^{-1}
\end{array}\right)
$$

if $W_{1} \leq 0$, or equivalently if for a sufficiently small $\alpha_{c}$ and some matrices $X_{c}=P_{c}$ and $Y_{c} \in \mathbb{R}^{1 \times n}$ the LMI

$$
\left(\begin{array}{cc}
A X_{c}+X_{c} A^{T}+b Y_{c}+Y_{c}^{T} b^{T}+\alpha_{c} X_{c} & b Y_{c} \\
Y_{c}^{T} b^{T} & -\delta_{c} X_{c}
\end{array}\right) \leq 0
$$

is feasible, then the time derivative of $V_{c}$ is upper bounded as

$$
\dot{V}_{c} \leq-\alpha_{c}\left(V_{c}-1\right) \text {. }
$$

Inequality (10) implies that the ellipsoid (4) with $\tilde{P}=X_{c}$ is an attractive ellipsoid for (1). To minimize the size of the ellipsoid the $\operatorname{tr}\left(X_{c}\right)$ is selected as the objective function, and the LMI (9) as the constraint set for the following optimization problem

$$
\begin{aligned}
& \operatorname{tr}\left(X_{c}\right) \rightarrow \min _{X_{c}, Y_{c}} \\
& \text { subject to }(9),
\end{aligned}
$$

the solution $\left(\hat{X}_{c}, \hat{Y}_{c}\right)$ is then used to calculate the minimizing control gain as $\hat{k}_{c}=\hat{Y}_{c} \hat{X}_{c}^{-1}$.

\section{Numerical Aspects}

The optimization problem presented in equation (8) is nonlinear, the LMI constraints ask for the maximum $\left(r_{1}\right)$ and minimum $\left(r_{2}\right)$ eigenvalues of the matrix variable $X$, as well as for some scalar variables $\delta$ and $\beta$. To solve this problem the following heuristic algorithm is proposed. 
We separate the algorithm in two linear optimization problems. First, let us fix some initial values $\left(r_{1}, r_{2}, \delta, \beta\right)$ such that the LMI (6) is feasible. Now, the optimization problem (8) is linear and has solution w.r.t the matrix variables $(X, Y)$. Denote this first optimization problem by SDPproblem 1 . Then, the values of $r_{1}$ and $r_{2}$ are updated with the obtained solution $\left(r_{1} \leftarrow 1 / \lambda_{\max }(X), \quad r_{2} \leftarrow\right.$ $\left.1 / \lambda_{\min }(X)\right)$. In order to reduce the settling time and guarantee the fulfillment of $(6)$, the parameter $\delta$ has to be maximized. For the next part the matrices $(X, Y)$ are fixed to the previous solution so the optimization problem

$$
\begin{aligned}
& \delta \rightarrow \max _{\delta, \beta} \\
& \text { subject to }(6)
\end{aligned}
$$

denoted by $S D P$ problem 2 , is now linear w.r.t. the scalars $\delta$ and $\beta$. This procedure is repeated until a certain precision condition is met or the solver is not able to find a solution anymore.

Note that the second inequality of (6) can be rewritten using the Schur's complement as

$$
\left(\begin{array}{cc}
X H_{\mu}+H_{\mu} X & \delta I_{n} \\
\delta I_{n} & X^{-1}
\end{array}\right) \geq 0
$$

so it is linear w.r.t $\delta$ for a fixed $X$. Also, note that the maximization of $\delta$ does not increase the size of the ellipsoid, in fact bigger values of $\delta$ would allow smaller values of $X$ in the corresponding diagonal term of matrix $\Omega$.

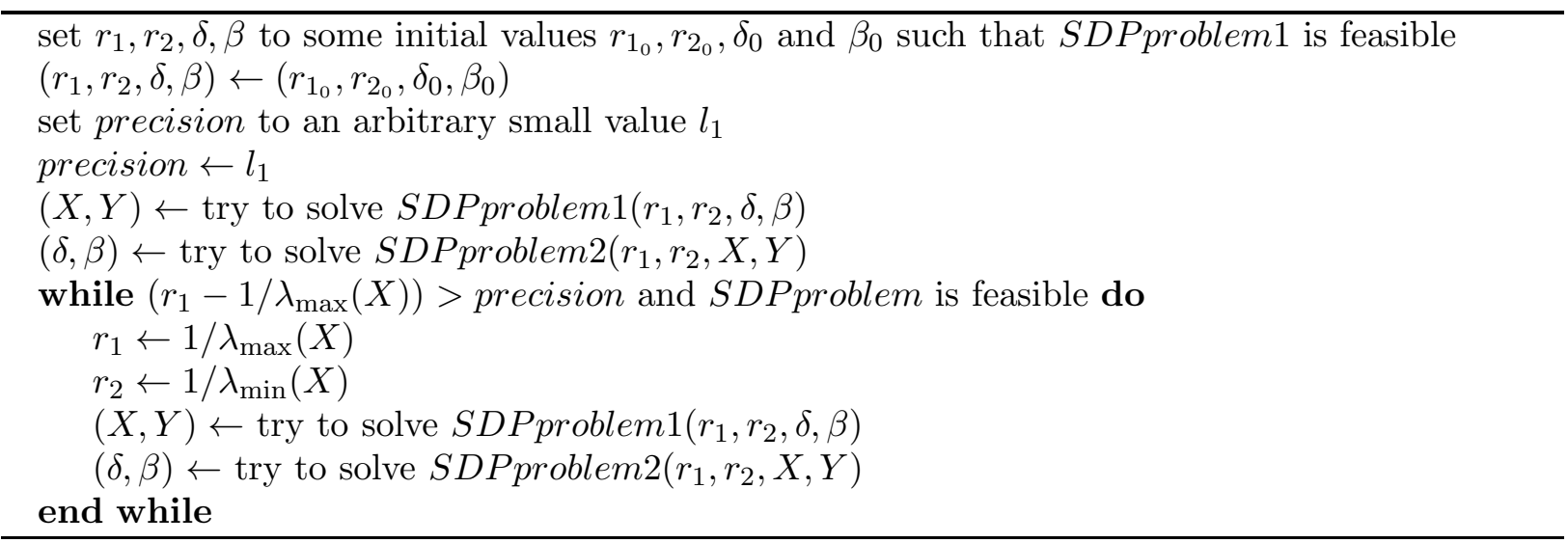

\subsection{Example}

Considering a chain of integrators as in (1) with $A \in \mathbb{R}^{3 \times 3}$, with the initial conditions $x_{0}=(1,1,1)^{T}$ the selected perturbation considered was $\xi(t)=0.5+0.25 \sin (100 t)+$ $0.25 \sin (t)(0.001, \quad 0.01, \quad 0.1)^{T}$, and the matrix $Q$ is defined as

$$
Q=\left(\begin{array}{ccc}
3.3333 & 0 & 0 \\
0 & 0.0333 & 0 \\
0 & 0 & 0.0003
\end{array}\right) \times 10^{5} .
$$

Selecting $\mu=0.5, \delta=1.8, \sigma^{2}=125$ and $\beta=1$, the ellipsoidal matrix and the matrix gain 
obtained from the solution of the LMI problem stated in theorem 3, are the following

$$
\begin{aligned}
\tilde{P} & =\left(\begin{array}{ccc}
0.0118 & -0.0104 & -0.0013 \\
-0.0104 & 0.0175 & -0.0116 \\
-0.0013 & -0.0116 & 0.1219
\end{array}\right), \\
\hat{k} & =\left(\begin{array}{lll}
-28.3424 & -31.6649 & -12.8397
\end{array}\right),
\end{aligned}
$$

and $\bar{\gamma}=5.1707$.

The function $V$ is calculated by the algorithm presented in (Polyakov et al., 2013) considering $V_{\min }=1$, which is based on the bisection numerical method to find the zeros of (5). The simulations have been done using the explicit Euler method with a sampling period with 0.001 seconds.

For the sake of comparison we include the results of the linear AEM applied to system (1). The ellipsoid matrix and the control gain obtained are the following

$$
\begin{aligned}
& \tilde{P}_{c}=\left(\begin{array}{lll}
0.0147 & 0.0060 & 0.0011 \\
0.0060 & 0.0683 & 0.0111 \\
0.0011 & 0.0111 & 0.0159
\end{array}\right), \\
& \hat{k}_{c}=\left(\begin{array}{lll}
-0.5102 & -2.4469 & -1.0640
\end{array}\right),
\end{aligned}
$$

Figures 1 and 2 show the comparison of the ellipsoids and system trajectories projected on the subspace $\left(x_{1}, x_{2}\right)$ and $\left(x_{3}, x_{2}\right)$ respectively for each approach. The next two figures, 3 and 4 show the states and control input comparison, and finally the figure 5 compares the ellipsoid and system trajectories in $\mathbb{R}^{3}$.

Remark 1: It is important to note that the faster convergence rate for the ILF approach is only assured locally, globally the linear case can be faster. This means that for initial conditions closer to the origin the ILF approach would be faster than the linear case, but for initial conditions chosen much farther from the origin the linear approach would be faster than the ILF approach.

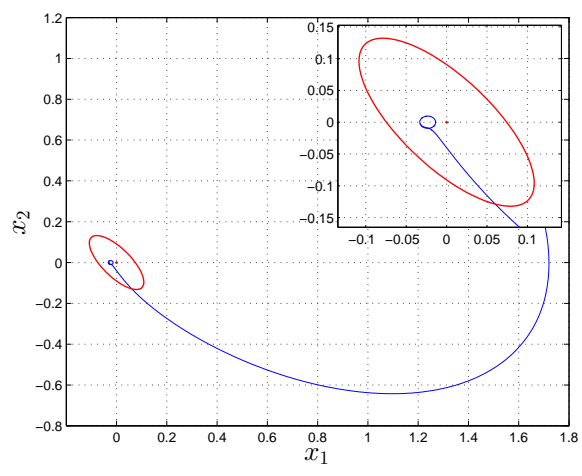

(a) ILF approach.

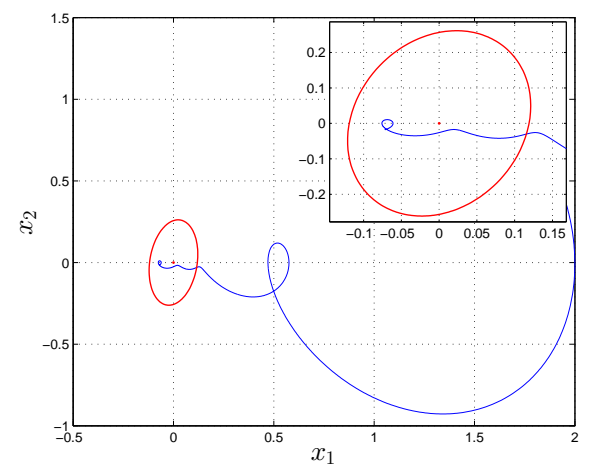

(b) Linear approach.

Figure 1.: Projection on the subspace $\left(x_{1}, x_{2}\right)$. 


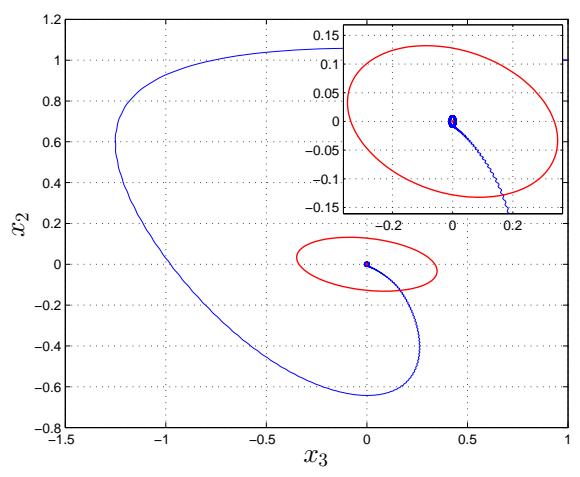

(a) ILF approach.

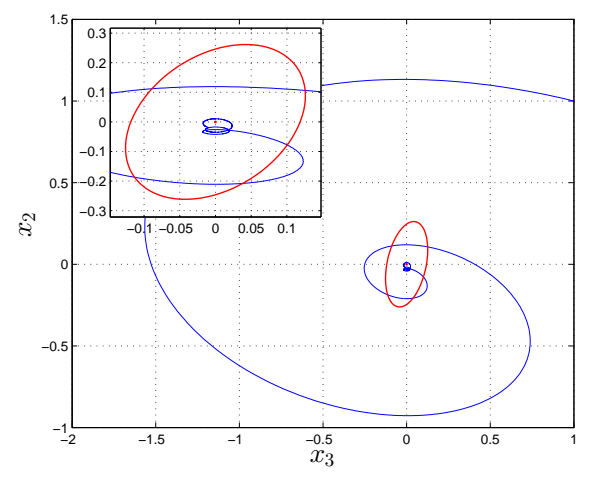

(b) Linear approach.

Figure 2.: Projection on the subspace $\left(x_{3}, x_{2}\right)$.

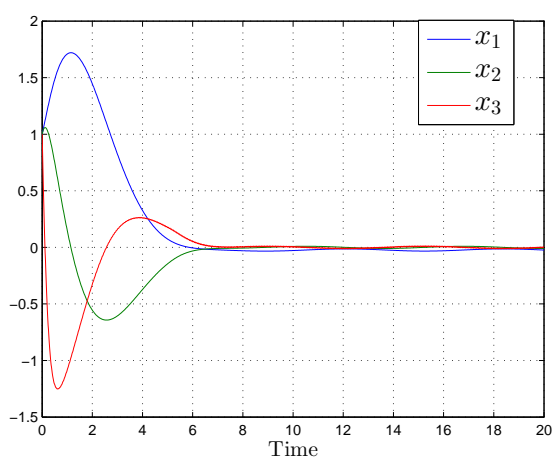

(a) ILF approach.

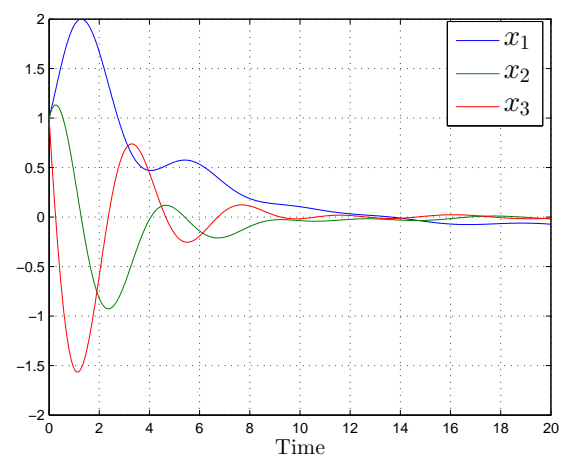

(b) Linear approach.

Figure 3.: States.

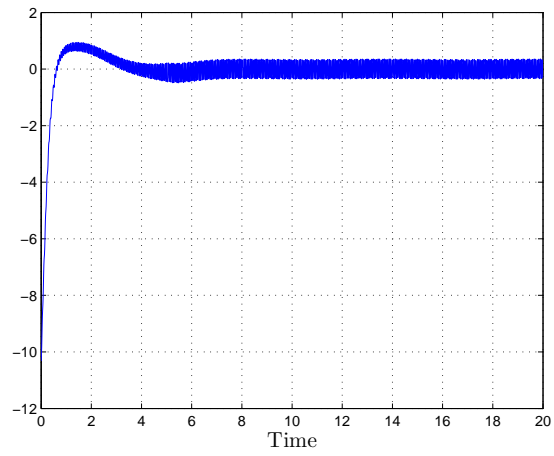

(a) ILF approach.

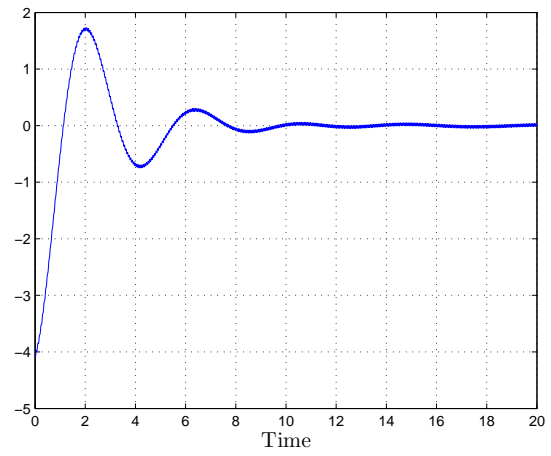

(b) Linear approach.

Figure 4.: Control.

\section{Conclusions}

A theoretical extension of the well known Attractive Ellipsoid Method was developed in this paper using the Implicit Lyapunov Function approach. Finite-time convergence despite noisy measurements to an ellipsoidal set is proved. This is achieved using a control input obtained from an ILF and the solution of a linear, respect to the matrix variables, minimization problem. The imple- 


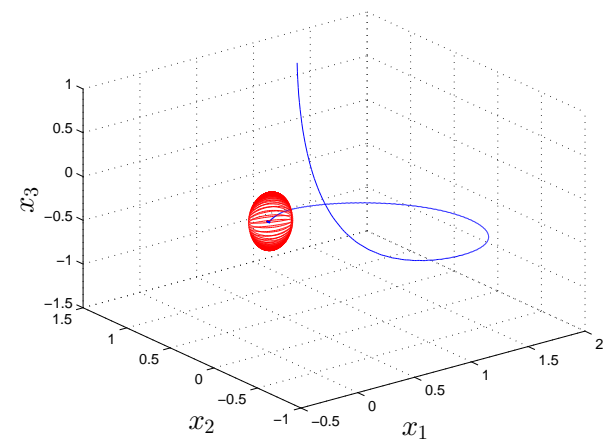

(a) ILF approach.

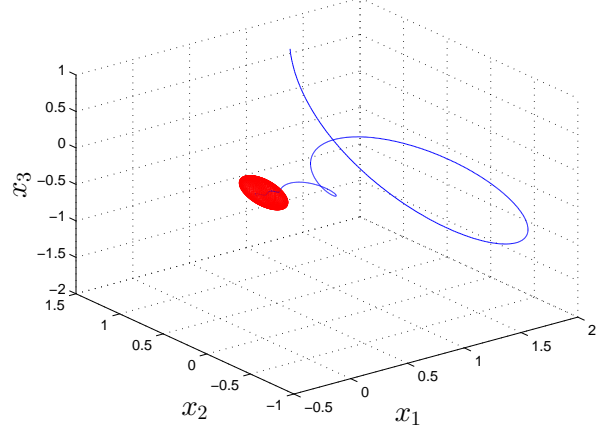

(b) Linear approach.

Figure 5.: Ellipsoid $\mathbb{R}^{3}$.

mentable numerical algorithm, based on the above extension is validated through an example, and compared with the results of a linear version of the AEM.

\section{Appendix}

\subsection{Supporting Facts}

Lemma 1: Considering $x$ defined as in (1), $\xi$ and $Q$ as in (2), and $G(V, x)$ as in (5). Let a matrix $P=P^{T}$ such that $0<P \leq Q$, and $(x+\xi)^{T} P(x+\xi)=1$. Then, if $G(V, x)=0, V$ is bounded as

$$
\underline{\gamma} \leq V \leq \bar{\gamma}
$$

with

$$
\underline{\gamma}=\sqrt{\frac{r_{1}}{r_{2}}}\left(1-\sqrt{\frac{r_{1}}{r_{3}}}\right), \bar{\gamma}=\sqrt{\frac{r_{2}}{r_{1}}}\left(1+\sqrt{\frac{r_{1}}{r_{3}}}\right),
$$

and $r_{1}:=\lambda_{\min }(P), r_{2}:=\lambda_{\max }(P)$ and $r_{3}:=\lambda_{\min }(Q)$.

Proof. If $G(V, x)=0$, this implies

$$
x^{T} D_{r}\left(V^{-1}\right) P D_{r}\left(V^{-1}\right) x=1 .
$$

Directly from there, some lower and upper bounds, for $x^{T} D_{r}\left(V^{-1}\right) P D_{r}\left(V^{-1}\right) x$, can be obtained as

$$
\frac{\lambda_{\min }(P)}{\lambda_{\max }(P)} \lambda_{\min }\left(D_{r}\left(V^{-1}\right)^{2}\right) \underline{g} \leq 1 \leq \frac{\lambda_{\max }(P)}{\lambda_{\min }(P)} \lambda_{\max }\left(D_{r}\left(V^{-1}\right)^{2}\right) \bar{g}
$$

where $\underline{g}$ and $\bar{g}$ are respectively the minimum and maximum values of $x^{T} P x$, subject to the following constraints

$$
\begin{aligned}
\text { minimize / maximize } & x^{T} P x \\
\text { subject to } & (x+\xi)^{T} P(x+\xi)=1, \quad \xi^{T} Q \xi \leq 1 .
\end{aligned}
$$


These optimization problems can be rewritten for simplicity sake using the following variables

$$
Y=P^{\frac{1}{2}}(x+\xi), \quad Z=-P^{\frac{1}{2}} \xi, W=P^{-\frac{1}{2}} Q P^{-\frac{1}{2}}
$$

as

$$
\begin{aligned}
\text { minimize/maximize } & (Y+Z)^{T}(Y+Z) \\
\text { subject to } & Y^{T} Y=1, Z^{T} W Z \leq 1 .
\end{aligned}
$$

To solve this quadratic minimization problem we first fix $Z$, and then we minimize $Y^{T} Y+2 Y^{T} Z+$ $Z^{T} Z$ w.r.t $Y$ with $Y^{T} Y=1$, the solution to this minimization problem can be shown to be $Y=-\frac{Z}{\|Z\|}$. Selecting $Y$ as this solution we minimize the remaining expression $(1-\|Z\|)^{2}$ w.r.t. $Z$, subject to the constraint $Z^{T} W Z \leq 1$,

$$
\begin{aligned}
& (1-\|Z\|)^{2} \rightarrow \min \\
& \text { subject to } \quad Z^{T} W Z \leq 1 .
\end{aligned}
$$

It is possible to make an additional change of variables $\tilde{Z}=W^{1 / 2} Z$ to rewrite this problem as

$$
\begin{aligned}
& \left(1-\left\|W^{-1 / 2} \tilde{Z}\right\|\right)^{2} \rightarrow \min \\
& \text { subject to }\|\tilde{Z}\|=1,
\end{aligned}
$$

knowing that

$$
\frac{1}{\sqrt{\lambda_{\max }(W)}} \leq \underset{\|\tilde{Z}\|=1}{-1 / 2} \tilde{Z} \| \leq \frac{1}{\sqrt{\lambda_{\min }(W)}}
$$

and particularly for our case, because

$$
P \leq Q \Rightarrow I \leq W \Rightarrow 1 \leq \lambda_{\min }(W) \leq \lambda_{\max }(W),
$$

then it is obvious that

$$
\begin{aligned}
\underline{g} & :=\min \left\{\left(1-\frac{1}{\sqrt{\lambda_{\min }(W)}}\right)^{2},\left(1-\frac{1}{\sqrt{\lambda_{\max }(W)}}\right)^{2}\right\} \\
& =\left(1-\frac{1}{\sqrt{\lambda_{\min }(W)}}\right)^{2},
\end{aligned}
$$

is the solution to the above quadratic minimization problem.

The maximization problem can be solved in the same way, and the solution can be shown to be

$$
\bar{g}:=\left(1+\frac{1}{\sqrt{\lambda_{\min }(W)}}\right)^{2} .
$$

Finally, taking into account that for the worst case when $V \leq 1$ the minimum value for $D_{r}\left(V^{-1}\right)^{2}$ is $\lambda_{\min }\left(D_{r}\left(V^{-1}\right)^{2}\right)=V^{-2}$. Analogously, for the worst case when $V \geq 1$, the maximum possible 
reached value would be $\lambda_{\max }\left(D_{r}\left(V^{-1}\right)^{2}\right)=V^{-2}$. Considering both cases, some upper and lower bounds for $V$ are

$$
\underline{\gamma}=\sqrt{\frac{\lambda_{\min }(P)}{\lambda_{\max }(P)}} \underline{g} \leq V \leq \sqrt{\frac{\lambda_{\max }(P)}{\lambda_{\min }(P)}} \bar{g}=\bar{\gamma} .
$$

\subsection{Proof of Theorem 3}

The function (5) satisfies the conditions C1-C3. The condition C4 also holds since $0<H_{\mu} P+P H_{\mu}$. We have

$$
0>\frac{\partial G}{\partial V}=-V^{-1} x^{T} D_{r}\left(V^{-1}\right)\left[P H_{\mu}+H_{\mu} P\right] D_{r}\left(V^{-1}\right) x
$$

and

$$
\frac{\partial G}{\partial x} \dot{x}=2 x^{T} D_{r}\left(V^{-1}\right) P D_{r}\left(V^{-1}\right)[A x+b u]
$$

To obtain finite-time convergence we apply the control (7)

$$
u\left(V_{y}, y\right):=V_{y}^{1-\mu} k D_{r}\left(V_{y}^{-1}\right) y=V_{y}^{1-\mu} k D_{r}\left(V_{y}^{-1}\right)[x+\xi],
$$

where the vector $k^{T} \in \mathbb{R}^{n}$ is the feedback gain. Substituting (7) in (13) and adding and subtracting $b V^{1-\mu} k D_{r}\left(V^{-1}\right) x$, we obtain

$$
\begin{aligned}
& \frac{\partial G}{\partial x} \dot{x}=2 x^{T} D_{r}\left(V^{-1}\right) P D_{r}\left(V^{-1}\right)\left[A x+b V_{y}^{1-\mu} k D_{r}\left(V_{y}^{-1}\right) x\right. \\
& \left.+b V_{y}^{1-\mu} k D_{r}\left(V_{y}^{-1}\right) \xi+b V^{1-\mu} k D_{r}\left(V^{-1}\right) x-b V^{1-\mu} k D_{r}\left(V^{-1}\right) x\right] \\
& =2 V^{-\mu} x^{T} D_{r}\left(V^{-1}\right) P D_{r}\left(V^{-1}\right)\left[V^{\mu} A x+b V k D_{r}\left(V^{-1}\right) x\right] \\
& +2 x^{T} D_{r}\left(V^{-1}\right) P D_{r}\left(V^{-1}\right)\left[b V_{y}^{1-\mu} k D_{r}\left(V_{y}^{-1}\right) x-b V^{1-\mu} k D_{r}\left(V^{-1}\right) x\right] \\
& +2 x^{T} D_{r}\left(V^{-1}\right) P D_{r}\left(V^{-1}\right)\left[b V_{y}^{1-\mu} k D_{r}\left(V_{y}^{-1}\right) \xi\right]
\end{aligned}
$$

using the obvious properties, of $G$ we know that

$$
\begin{aligned}
& V^{\mu} A=\left[D_{r}\left(V^{-1}\right)\right]^{-1} A D_{r}\left(V^{-1}\right), \\
& V b k=\left[D_{r}\left(V^{-1}\right)\right]^{-1} b k,
\end{aligned}
$$


we can write

$$
\begin{aligned}
& \frac{\partial G}{\partial x} \dot{x}=2 V^{-\mu} x^{T} D_{r}\left(V^{-1}\right)[P A+P b k] D_{r}\left(V^{-1}\right) x \\
& +2 x^{T} D_{r}\left(V^{-1}\right) P D_{r}\left(V^{-1}\right) b k\left[V_{y}^{1-\mu} D_{r}\left(V_{y}^{-1}\right)-V^{1-\mu} D_{r}\left(V^{-1}\right)\right] x \\
& +2 x^{T} D_{r}\left(V^{-1}\right) P D_{r}\left(V^{-1}\right) b V_{y}^{1-\mu} k D_{r}\left(V_{y}^{-1}\right) \xi \\
& =V^{-\mu}\left(2 x^{T} D_{r}\left(V^{-1}\right)[P A+P b k] D_{r}\left(V^{-1}\right) x\right. \\
& +2 V^{\mu} x^{T} D_{r}\left(V^{-1}\right) P D_{r}\left(V^{-1}\right) b k\left[V_{y}^{1-\mu} D_{r}\left(V_{y}^{-1}\right)-V^{1-\mu} D_{r}\left(V^{-1}\right)\right] x \\
& \left.+2 \varepsilon^{1-\mu} x^{T} D_{r}\left(V^{-1}\right) P b k D_{r}\left(V_{y}^{-1}\right) \xi\right)
\end{aligned}
$$

with $\varepsilon:=\frac{V_{y}}{V}$. Now, using property (14) and considering $\left[D_{r}\left(V^{-1}\right)\right]^{-1} D_{r}\left(V^{-1}\right)=I_{n}$, the second term can be rewritten as follows

$$
\begin{aligned}
& 2 V^{\mu} x^{T} D_{r}\left(V^{-1}\right) P D_{r}\left(V^{-1}\right) b k\left[V_{y}^{1-\mu} D_{r}\left(V_{y}^{-1}\right)\right. \\
& \left.-V^{1-\mu} D_{r}\left(V^{-1}\right)\right] x \\
= & 2 V^{\mu} x^{T} D_{r}\left(V^{-1}\right) P D_{r}\left(V^{-1}\right) b V V^{-1} k\left[V_{y}^{1-\mu} D_{r}\left(V_{y}^{-1}\right)\right. \\
& \left.\left.-V^{1-\mu} D_{r}\left(V^{-1}\right)\right]\left[D_{r}\left(V^{-1}\right)\right]^{-1} D_{r}\left(V^{-1}\right)\right] x \\
= & 2 x^{T} D_{r}\left(V^{-1}\right) P b k\left(\varepsilon^{1-\mu} D_{r}\left(V_{y}^{-1}\right)\right. \\
& \left.-D_{r}\left(V^{-1}\right)\right)\left[D_{r}\left(V^{-1}\right)\right]^{-1} D_{r}\left(V^{-1}\right) x \\
= & 2 x^{T} D_{r}\left(V^{-1}\right) P b k \tilde{D}_{r}\left(\varepsilon^{-1}\right) D_{r}\left(V^{-1}\right) x
\end{aligned}
$$

where $\tilde{D}_{r}\left(\varepsilon^{-1}\right):=\operatorname{diag}\left\{\varepsilon^{-(n-i+1) \mu}-1\right\}_{i=1}^{n}$. After all these manipulations (15) looks as

$$
\begin{aligned}
\frac{\partial G}{\partial x} \dot{x}= & V^{-\mu}\left(2 x^{T} D_{r}\left(V^{-1}\right)\left[P A+P b k+P b k \tilde{D}_{r}\left(\varepsilon^{-1}\right)\right] D_{r}\left(V^{-1}\right) x\right. \\
& \left.+2 \varepsilon^{1-\mu} x^{T} D_{r}\left(V^{-1}\right) P b k D_{r}\left(V_{y}^{-1}\right) \xi\right)
\end{aligned}
$$

and introducing the extended vector

$$
z=\left(\begin{array}{c}
D_{r}\left(V^{-1}\right) x \\
D_{r}\left(V_{y}^{-1}\right) \xi
\end{array}\right)
$$

the last expression can be rewritten in compact form as

$$
\begin{aligned}
\frac{\partial G}{\partial x} \dot{x} & =V^{-\mu}\left(z^{T} \Omega_{0} z+2 x^{T} D_{r}\left(V^{-1}\right) P b k \tilde{D}_{r}\left(\varepsilon^{-1}\right) D_{r}\left(V^{-1}\right) x\right) \\
\Omega_{0} & =\left(\begin{array}{cc}
P A+A^{T} P+P b k+k^{T} b^{T} P & \varepsilon^{1-\mu} P b k \\
\varepsilon^{1-\mu} k^{T} b^{T} P & 0
\end{array}\right) .
\end{aligned}
$$

Now, adding and subtracting the following terms from (16),

$$
\begin{aligned}
& V^{-\mu} x^{T} D_{r}\left(V^{-1}\right)\left[H_{\mu} P+P H_{\mu}\right] D_{r}\left(V^{-1}\right) x \\
& V^{-\mu} \delta \xi^{T} D_{r}\left(V_{y}^{-1}\right) Q D_{r}\left(V_{y}^{-1}\right) \xi
\end{aligned}
$$


where $\delta \in \mathbb{R}_{+}$is a constant. It becomes

$$
\begin{aligned}
\frac{\partial G}{\partial x} \dot{x}= & V^{-\mu}\left(z^{T} \Omega_{1} z-x^{T} D_{r}\left(V^{-1}\right)\left[H_{\mu} P+P H_{\mu}\right] D_{r}\left(V^{-1}\right) x\right. \\
& +2 x^{T} D_{r}\left(V^{-1}\right) P b k \tilde{D}_{r}\left(\varepsilon^{-1}\right) D_{r}\left(V^{-1}\right) x \\
& \left.+\delta \xi^{T} D_{r}\left(V_{y}^{-1}\right) Q D_{r}\left(V_{y}^{-1}\right) \xi\right), \\
\Omega_{1}= & \left(\begin{array}{cc}
P A+A^{T} P+P b k+k^{T} b^{T} P & \varepsilon^{1-\mu} P b k \\
+H_{\mu} P+P H_{\mu} & -\delta Q
\end{array}\right) .
\end{aligned}
$$

Using the $\Lambda$-inequality (Poznyak, 2008) $\left(\mathcal{X}^{\top} \mathcal{Y}+\mathcal{Y}^{\top} \mathcal{X} \leq \mathcal{X}^{\top} \Lambda \mathcal{X}+\mathcal{Y}^{\top} \Lambda^{-1} \mathcal{Y}\right)$ on the term involving $\varepsilon$ and setting $\Lambda=\beta^{-1} P^{-1}, \mathcal{X}=k^{T} b^{T} P$, and $\mathcal{Y}=\tilde{D}_{r}\left(\varepsilon^{-1}\right)$, where $\beta \in \mathbb{R}_{+}$

$$
2 P b k \tilde{D}_{r}\left(\varepsilon^{-1}\right) \leq \beta^{-1}(P b k) P^{-1}\left(k^{T} b^{T} P\right)+\beta \tilde{D}_{r}\left(\varepsilon^{-1}\right) P \tilde{D}_{r}\left(\varepsilon^{-1}\right) .
$$

Consequently,

$$
\begin{gathered}
\frac{\partial G}{\partial x} \dot{x} \leq V^{-\mu}\left(z^{T} \Omega_{2} z-x^{T} D_{r}\left(V^{-1}\right)\left[H_{\mu} P+P H_{\mu}\right] D_{r}\left(V^{-1}\right) x\right. \\
+\delta \xi^{T} D_{r}\left(V_{y}^{-1}\right) P D_{r}\left(V_{y}^{-1}\right) \xi \\
\left.+\beta^{-1} x^{T} D_{r}\left(V^{-1}\right)(P b k) P^{-1}\left(k^{T} b^{T} P\right) D_{r}\left(V^{-1}\right) x\right), \\
\Omega_{2}=\left(\begin{array}{cc}
P A+A^{T} P+P b k+k^{T} b^{T} P+H_{\mu} P+P H_{\mu} & \varepsilon^{1-\mu} P b k \\
+\beta \tilde{D}_{r}\left(\varepsilon^{-1}\right) P \tilde{D}_{r}\left(\varepsilon^{-1}\right) & -\delta Q \\
\varepsilon^{1-\mu} k^{T} b^{T} P &
\end{array}\right.
\end{gathered}
$$

applying the Schur's complement it can be shown that

$$
\begin{aligned}
\frac{\partial G}{\partial x} \dot{x} \leq & -V^{-\mu} x^{T} D_{r}\left(V^{-1}\right)\left[H_{\mu} P+P H_{\mu}\right] D_{r}\left(V^{-1}\right) x \\
& +\delta V^{-\mu} \xi^{T} D_{r}\left(V_{y}^{-1}\right) Q D_{r}\left(V_{y}^{-1}\right) \xi
\end{aligned}
$$

if

$$
\left(\begin{array}{ccc}
P(A+b k)+(A+b k)^{T} P & & \\
+H_{\mu} P+P H_{\mu}+\beta \tilde{D}_{r}\left(\varepsilon^{-1}\right) P \tilde{D}_{r}\left(\varepsilon^{-1}\right) & P b k & P b k \\
k^{T} b^{T} P & -\varepsilon^{-2+2 \mu} \delta Q & 0 \\
k^{T} b^{T} P & 0 & -\beta P
\end{array}\right) \leq 0 .
$$

However, to assure the semi-definite negativeness of the last matrix first it is necessary to find some suitable bounds for $\varepsilon$.

Using Lemma 1, analyzing the case when the system trajectories fulfill $V_{y}=1$ and, next applying the homogeneous dilation it is possible to find sufficient conditions for the parameters of (17) such that finite-time convergence of the system trajectories to the ellipsoid $\mathcal{E}_{x}$ is assured. If $V_{y}=1$ then for $\varepsilon^{-1}$ we would have $\varepsilon^{-1}=V$, and directly from Lemma 1

$$
\underline{\gamma} \leq \varepsilon^{-1} \leq \bar{\gamma}
$$


Then, $-\varepsilon^{-2+2 \mu} \leq-\underline{\gamma}^{-2+2 \mu}$, and considering that $r_{1}=\lambda_{\min }(P)$, we can define

$$
m_{i}:=\max \left\{\left|\underline{\gamma}^{-(n-i+1) \mu}-1\right|,\left|\bar{\gamma}^{-(n-i+1) \mu}-1\right|\right\}, \text { for each } i,
$$

such that the square of the matrix $\tilde{D}_{m}=\operatorname{diag}\left\{m_{i}\right\}_{i=1}^{n}$, is a bound for the term involving $\tilde{D}_{r}\left(\varepsilon^{-1}\right)$ as

$$
\tilde{D}_{r}\left(\varepsilon^{-1}\right) P \tilde{D}_{r}\left(\varepsilon^{-1}\right) \leq \frac{1}{r_{1}} \tilde{D}_{r}\left(\varepsilon^{-1}\right)^{2} \leq \frac{1}{r_{1}} \tilde{D}_{m}^{2}
$$

with this bound, applying the Schur's complement one more time and using the fact that $P \leq Q$, the next inequality is obtained

$$
\Omega=\left(\begin{array}{cccc}
P(A+b k)+(A+b k)^{T} P & P b k & P b k & \beta I_{n} \\
+H_{\mu} P+P H_{\mu} & -\underline{\gamma}^{-2+2 \mu} \delta P & 0 & 0 \\
k^{T} b^{T} P & 0 & -\beta P & 0 \\
k^{T} b^{T} P & 0 & 0 & -r_{1} \beta \tilde{D}_{m}^{-2}
\end{array}\right) \leq 0
$$

if the last inequality holds, then (17) holds.

For the term involving $\xi$ we consider any point belonging to the region defined by $V_{y}=1$, thus

$$
\xi^{T} D_{r}\left(V_{y}^{-1}\right) Q D_{r}\left(V_{y}^{-1}\right) \xi=\xi^{T} Q \xi
$$

and the derivative with respect to $V_{y}$

$$
\begin{aligned}
& \frac{\partial}{\partial V_{y}} \xi^{T} D_{r}\left(V_{y}^{-1}\right) Q D_{r}\left(V_{y}^{-1}\right) \xi \\
& =-V_{y}^{-1} \xi^{T} D_{r}\left(V_{y}^{-1}\right)\left[Q H_{\mu}+H_{\mu} Q\right] D_{r}\left(V_{y}^{-1}\right) \xi<0 .
\end{aligned}
$$

Therefore, this ensures that for all $V_{y} \geq 1, \xi^{T} D_{r}\left(V_{y}^{-1}\right) Q D_{r}\left(V_{y}^{-1}\right) \xi$ is a monotonic decreasing function of $V_{y}$, and

$$
\xi^{T} D_{r}\left(V_{y}^{-1}\right) Q D_{r}\left(V_{y}^{-1}\right) \xi \leq \xi^{T} Q \xi \leq 1
$$

so $\frac{\partial G}{\partial x} \dot{x}$ is upper bounded as

$$
\frac{\partial G}{\partial x} \dot{x} \leq V^{-\mu}\left(-x^{T} D_{r}\left(V^{-1}\right)\left[H_{\mu} P+P H_{\mu}\right] D_{r}\left(V^{-1}\right) x+\delta\right)
$$

Inequalities (12) and (18) prove that $\varlimsup_{t \rightarrow \infty} V_{y} \leq 1$. From Lemma 1 we know that the maximum value of $V$ when $V_{y}=1$ is $\bar{\gamma}$. The next step is to select an adequate $\tilde{P}$ (i.e. the smallest $\tilde{P}$ that completely contains the asymptotically attractive set estimated by $\left.\varlimsup_{t \rightarrow \infty} V \leq \bar{\gamma}\right)$.

From the definition of $G$ we know that the ellipsoidal set $\mathcal{E}_{x}$ with $\tilde{P}^{-1}=D_{r}\left(\bar{\gamma}^{-1}\right) P D_{r}\left(\bar{\gamma}^{-1}\right)$, coincides with the level set $V(x) \leq \bar{\gamma}$ which we have already shown to be an asymptotically attractive set of (1). Then

$$
x^{T} D_{r}\left(\bar{\gamma}^{-1}\right) P D_{r}\left(\bar{\gamma}^{-1}\right) x \leq 1,
$$

is an attractive ellipsoid for system (1), with the control input (7). 
Finally, if $G(V, x)=0$ the time derivative of the Lyapunov function can be estimated as

$$
\begin{aligned}
\dot{V} & =-\left[\frac{\partial G}{\partial V}\right]^{-1} \frac{\partial G}{\partial x} \dot{x} \\
& \leq \frac{-x^{T} D_{r}\left(V^{-1}\right)\left[H_{\mu} P+P H_{\mu}\right] D_{r}\left(V^{-1}\right) x+\delta}{x^{T} D_{r}\left(V^{-1}\right)\left[H_{\mu} P+P H_{\mu}\right] D_{r}\left(V^{-1}\right) x} V^{1-\mu} \\
& =\left(-1+\frac{\delta x^{T} D_{r}\left(V^{-1}\right) P D_{r}\left(V^{-1}\right) x}{x^{T} D_{r}\left(V^{-1}\right)\left[H_{\mu} P+P H_{\mu}\right] D_{r}\left(V^{-1}\right) x}\right) V^{1-\mu} \\
& \leq\left(-1+\frac{\delta x^{T} D_{r}\left(V^{-1}\right) P D_{r}\left(V^{-1}\right) x}{\delta^{2} x^{T} D_{r}\left(V^{-1}\right) P D_{r}\left(V^{-1}\right) x}\right) V^{1-\mu}=-\left(1-\delta^{-1}\right) V^{1-\mu},
\end{aligned}
$$

so the ellipsoid (19) is also a finite-time attractive ellipsoid for the system (1), and the settling time function is estimated as

$$
T\left(x_{0}\right) \leq \frac{1}{\mu\left(1-\delta^{-1}\right)}\left(V^{\mu}\left(x_{0}\right)-\bar{\gamma}^{\mu}\right),
$$

because it is not possible to compute directly $V\left(x_{0}\right)$, we need to use $V\left(y_{0}\right)$ to approximate it. We know that $V\left(x_{0}\right) \leq \bar{\gamma} V\left(y_{0}\right)$, then for $T\left(x_{0}\right)$ we have

$$
T\left(x_{0}\right) \leq \frac{1}{\mu\left(1-\delta^{-1}\right)} \bar{\gamma}^{\mu}\left(V^{\mu}\left(y_{0}\right)-1\right) .
$$

\section{References}

Adamy, J., \& Flemming, A. (2004). Soft variable-structure controls: a survey. Automatica, 40, 1821-1844.

Bertsekas, D., \& Rhodes, I. (1971, Apr). Recursive state estimation for a set-membership description of uncertainty. Automatic Control, IEEE Transactions on, 16(2), 117-128.

Bhat, S., \& Bernstein, D. (2000). Finite time stability of continuous autonomous systems. SIAM Journal of Control and Optimization, 38(3), 751-766.

Chernousko, F. (1994). State estimation for dynamic systems. Boca Raton: CRC Press.

Chernousko, F., Ananevskii, I., \& Reshmin, S. (2008). Control of nonlinear dynamical systems: methods and applications. Springer-Verlag.

Courant, R., \& John, F. (2000). Introduction to calculus and analysis (vol. i/ii). Springer.

Gonzalez-Garcia, S., Polyakov, A., \& Poznyak, A. (2009). Output linear controller for a class of nonlinear systems using the invariant ellipsoid technique. In American control conference, 2009. (p. 1160-1165).

Haimo, V. (1986). Finite time controllers. SIAM Journal of Control and Optimization, 24(4), 760-770.

Haykin, S. (2009). Neural networks and learning machines. Prentice Hall.

Isidori, A. (1995). Nonlinear control systems. Springer.

Korobov, V. I. (1979). A general approach to the solution of the bounded control synthesis problem in a controllability problem. Mat. Sb., 109(4), 582-606.

Korobov, V. I. (2007). Controllability function method. M.Izhevsk: NIC Regularnaya i Haoticheskaya Dinamika, Computer Researches Institute.

Kurzhanskii, A. B. (1977). Control and observation under uncertainty (in russian). Moscow.

Levant, A. (2005, May). Homogeneity approach to high-order sliding mode design. Automatica, 41(5), 823 -830 .

Mera, M., Poznyak, A., Azhmyakov, V., \& Fridman, E. (2009, Jan). Robust control for a class of continuoustime dynamical systems with sample-data outputs. In Electrical engineering, computing science and automatic control,cce,2009 6th international conference on (p. 1-7). 
Moulay, E., \& Perruquetti, W. (2006). Finite time stability and stabilization of a class of continuous systems. Journal of Mathematical Analysis and Applications, 323(2), 1430 - 1443.

Orlov, Y. (2005). Finite time stability and robust control synthesis of uncertain switched systems. SIAM Journal of Control and Optimization, 43(4), 1253 - 1271.

Orlov, Y., \& Aguilar, L. (2014). Advanced $H_{\infty}$ Control. Birkhauser.

Perruquetti, W., Floquet, T., \& Moulay, E. (2008). Finite-time observers: application to secure communication. IEEE Transactions on Automatic Control, 53(1), 356-360.

Polyak, B. T., Nazin, S., Durieu, C., \& Walter, E. (2004). Ellipsoidal parameter or state estimation under model uncertainty. Automatica, 40, $1171-1179$.

Polyak, B. T., \& Topunov, M. V. (2008). Suppression of bounded exogenous disturbances: Output feedback. Automation and Remote Control, 69, $801-818$.

Polyakov, A., Efimov, D., \& Perruquetti, W. (2013). Finite-time stabilization using implicit Lyapunov function technique. In 9th symposium on nonlinear control systems (p. 140-145).

Polyakov, A., Efimov, D., \& Perruquetti, W. (2015). Finite-time and fixed-time stabilization: Implicit Lyapunov function approach. Automatica, 51, 332 - 340.

Poznyak, A. (2008). Advanced mathematical tools for automatic control engineers: Deterministic techniques. Amsterdam: Elsevier.

Poznyak, A., Azhmyakov, V., \& Mera, M. (2011). Practical output feedback stabilisation for a class of continuous-time dynamic systems under sample-data outputs. International Journal of Control, $84(8), 1408-1416$.

Poznyak, A., Polyakov, A., \& Azhmyakov, V. (2014). Attractive ellipsoids in robust control. Springer.

Roxin, E. (1966). On finite stability in control systems. Rendiconti del Circolo Matematico di Palermo, $15(3), 273-283$.

Schweppe, F. (1968, Feb). Recursive state estimation: Unknown but bounded errors and system inputs. Automatic Control, IEEE Transactions on, 13(1), 22-28.

Shtessel, Y., Edwards, C., Fridman, L., \& Levant, A. (2014). Sliding mode control and observation. Springer New York.

Usoro, P., Schweppe, F., Wormley, D., \& Gould, L. (1981). Ellipsoidal set-theoretic control synthesis. Journal of Dynamical Systems, Measurements, and Control, $104(4), 331-336$.

Utkin, V., Guldner, J., \& Shi, J. (2009). Sliding mode control in electro-mechanical systems. CRC Press.

Zubov, V. (1964). Methods of a.m. Lyapunov and their applications. Noordhoff. 